ORNL/SPR-2018/873

CRADA/NFE-16-06392

\title{
Scalable processing of ZnS nanoparticles for high photoluminescence efficiency quantum dots
}

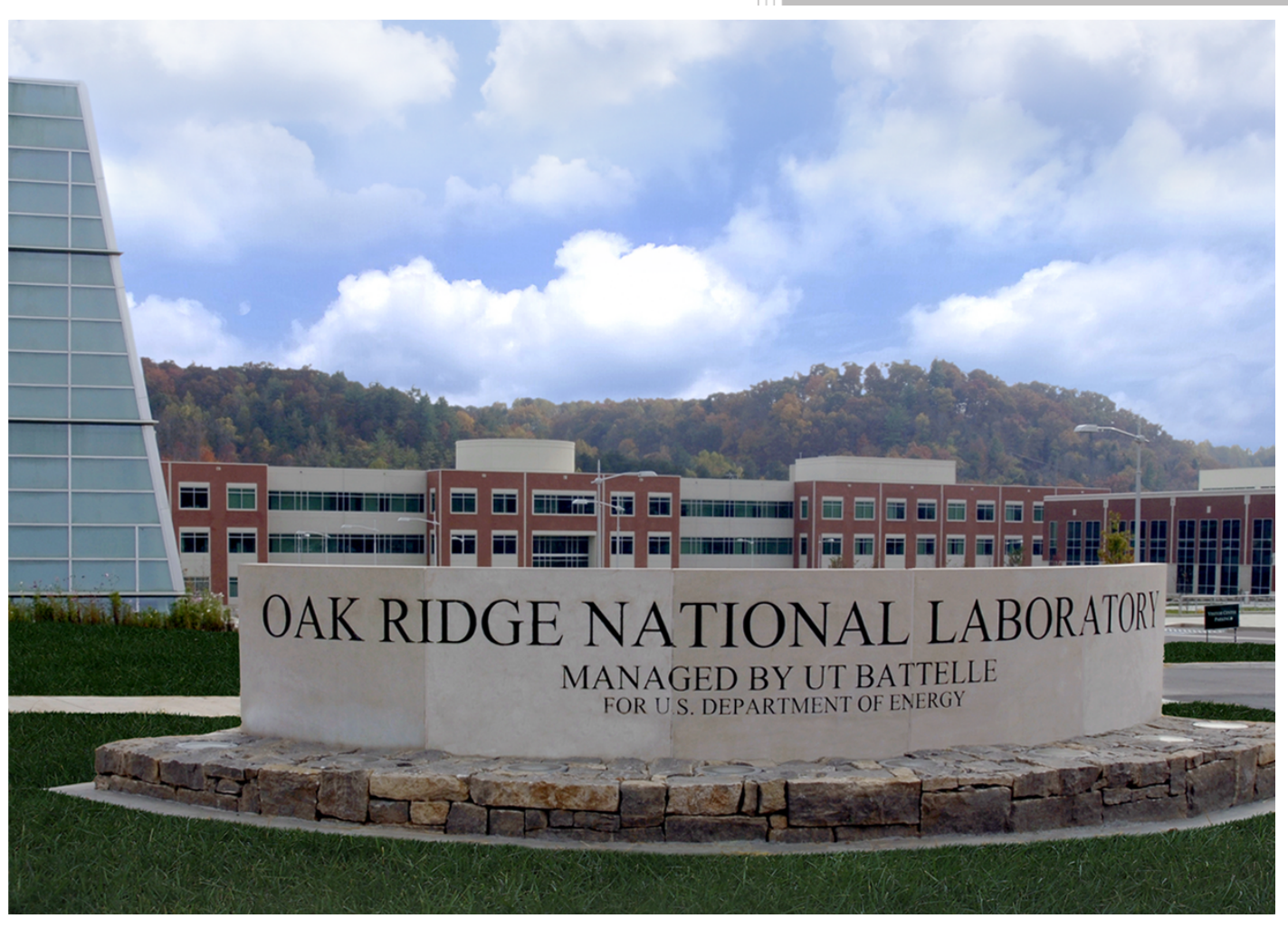

Kevin T. Scholtes

Christopher B. Jacobs

Eric E. Muckley

Patrick Caveney

Approved for public release;

Distribution is unlimited

Ilia N. Ivanov

April 24, 2018 


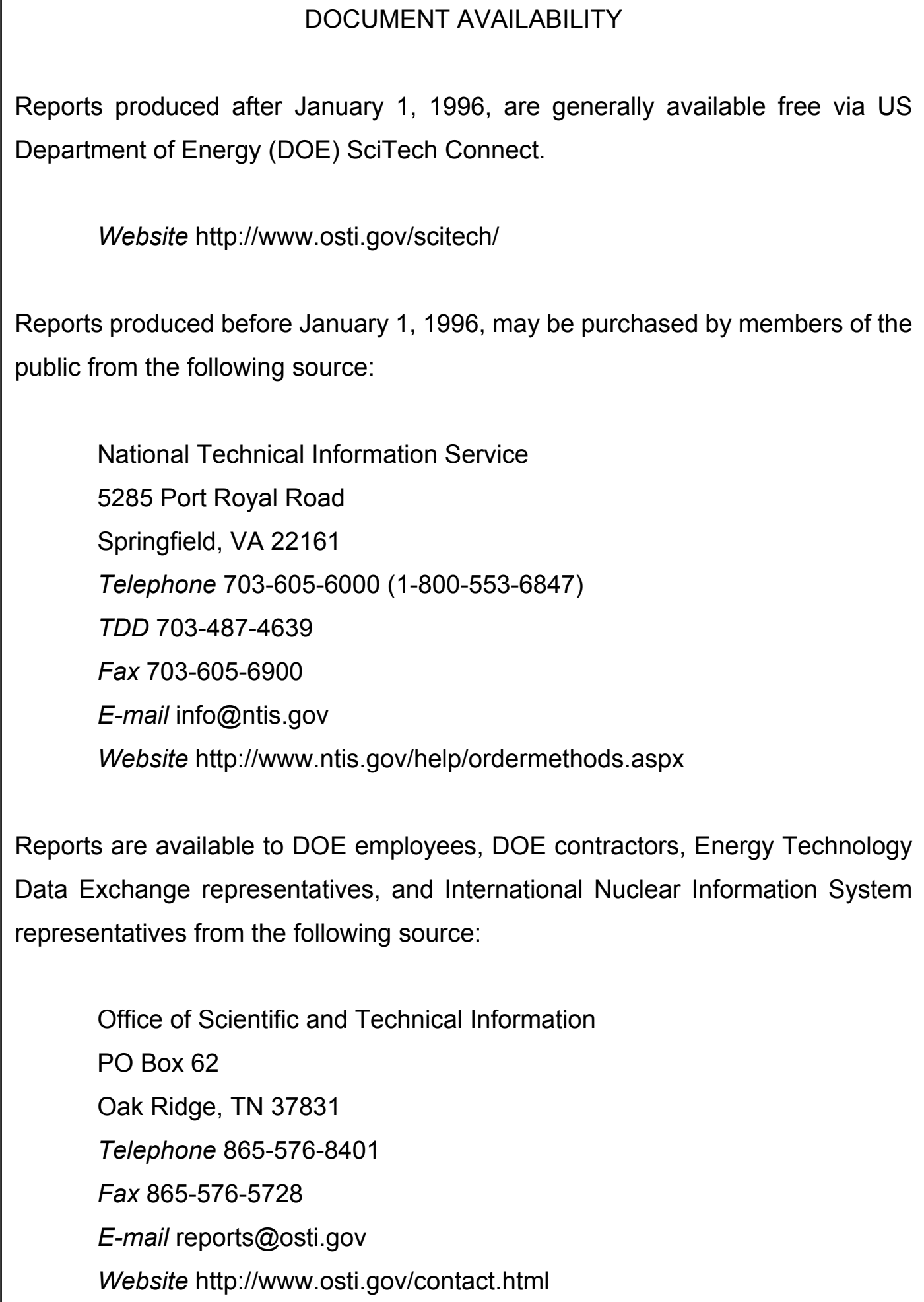


This report was prepared as an account of work sponsored by an agency of the United States Government. Neither the United States Government nor any agency thereof, nor any of their employees, makes any warranty, express or implied, or assumes any legal liability or responsibility for the accuracy, completeness, or usefulness of any information, apparatus, product, or process disclosed, or represents that its use would not infringe privately owned rights. Reference herein to any specific commercial product, process, or service by trade name, trademark, manufacturer, or otherwise, does not necessarily constitute or imply its endorsement, recommendation, or favoring by the United States Government or any agency thereof. The views and opinions of authors expressed herein do not necessarily state or reflect those of the United States Government or any agency thereof. 
Small Business Voucher Pilot

Technical Assistance from Oak Ridge National Laboratory to Nano Elements Source, LLC

\section{SCALABLE PROCESSING OF ZNS NANOPARTICLES FOR HIGH PHOTOLUMINESCENCE EFFICIENCY QUANTUM DOTS}

Kevin T. Scholtes, Christopher B. Jacobs

Eric E. Muckley, Patrick Caveney, Ilia N. Ivanov

Date Published:

April 24, 2018

Prepared by

OAK RIDGE NATIONAL LABORATORY

Oak Ridge, Tennessee 37831-6283

managed by

UT-BATTELLE, LLC

for the

US DEPARTMENT OF ENERGY

under contract DE-AC05-00OR22725

Approved for

Public release; distribution is unlimited 


\section{Contents}

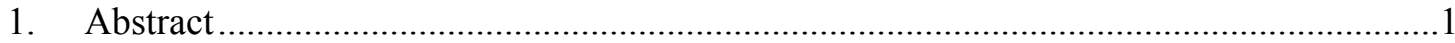

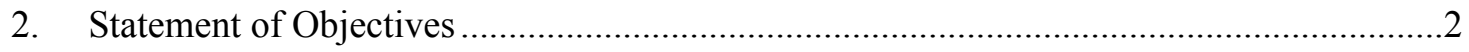

3. Benefits to the Funding DOE Office's Mission ...............................................................

4. Technical Discussion of Work Performed by All Parties ......................................................

4.1 Chemical passivation of surface defects .................................................................

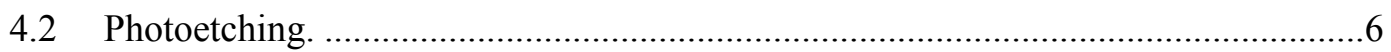

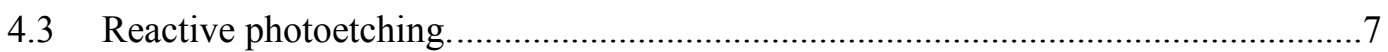

4.4 Control of the surface defect density in low temperature synthesis of $\mathrm{ZnS}$

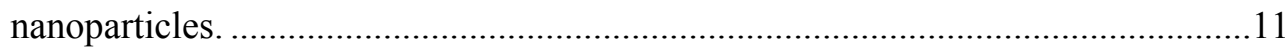

4.5 Investigation of the stability of chemically synthesized $\mathrm{ZnS}$ nanoparticles ............15

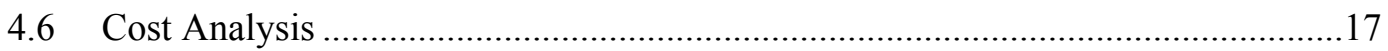

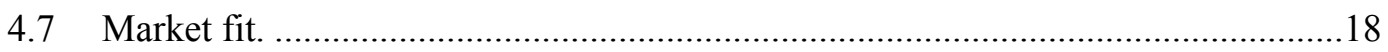

4.8 Commercialization Possibilities.........................................................................19

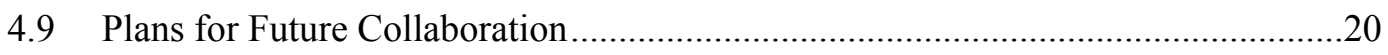

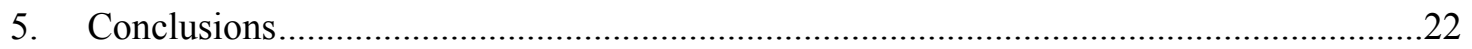

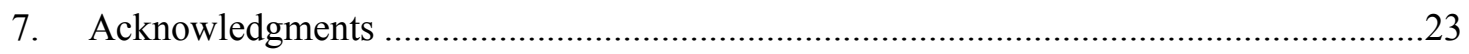




\section{ABSTRACT}

Nano Elements Source, LLC, a biotech startup has licensed the NanoFermentation technology from Oak Ridge National Laboratory to further commercialize low cost, cadmium free photoluminescent nanoparticles produced using affordable, environmentally-friendly. This project aimed to develop post chemical processing of biosynthesized $\mathrm{ZnS}$ quantum dots (QD) to improve their efficiency of luminescence to make them suitable for solid state lighting applications. Several post processing approached were proposed and tested using to biosynthesized of $\mathrm{ZnS}$ QD. Optical properties of $\mathrm{ZnS}$ nanoparticles including soft chemical etching, and photonic etching have been tested and quantified against standard materials. We applied the developed chemical modification approach to control quality of chemically synthetized ZnS QD's, and to calculate the maximum achievable efficiency of photoluminescence. We estimated the cost of the proposed post-processing treatment of nanoFermented nanoparticles and proposed several approaches to further the development of low cost nanoparticles for electro-optical and other applications relevant to DOE mission. 


\section{STATEMENT OF OBJECTIVES}

The goal of this project is to develop a solution allowing the NanoFermentation technology to move to mass production of nanoparticles to TRL--4-5. The current quality (low fluorescence efficiency) of nanoparticles produced through this process is not competitive with the targets sets by current BTO and SSL programs at EERE. The goal of the SBV funded research and development effort is to develop advanced low-cost post processing of NanoFermentation nanoparticles and demonstrate high photoluminescence quantum efficiency (PL QE) of $\mathrm{ZnS}$. We plan to further the development of this new technology by application to high PL QE of ternary, tertiary and core-shell nanoparticles through BTO and SSL programs. The results of R\&D efforts funded through the SBV program will serve as an example, proof-of-principle for new proposals. It will also access economic structure and cost of posttreatment. The SBV provides access to expertise and instrumentation, which is unavailable in the private sector. 


\section{BENEFITS TO THE FUNDING DOE OFFICE'S MISSION}

The need for economical and quality materials is a growing concern as technology is becoming more complex and difficult to manufacture. Nanoparticle quantum dots (QD) offer possible alternative solutions for electronics, optoelectronics and medical imaging. Due to their high luminescent efficiency and tunable band gap, making scalable and cost effective QD's is optimal in today's marketplace, especially for lighting and display applications. Methods currently used to generate QDs utilize high

temperature and multi-step processing schemes to yield high luminescent, but high-cost QD's. ${ }^{(13)}$ Low temperature synthesis methods such as sonication, UV irradiation and surfactants have the potential to reduce energy and labor costs for labs and commercial manufactures. Such development may improve the growth and advancement of green chemistry methods, safer businesses practices and efficient material fabrication.

In addition to energy savings gained from implementing QDs, there has been expressed government interest in improving the QD and QD Solid State Lighting markets. This research will further two specific goals of EERE's BTO: Emerging Technologies Program (by 2020, reduce building energy use 30\%), and Commercial Buildings Integration Plan (by 2025, cut energy use of market leaders by $35 \%$ ). These energy savings, through commercialization of biosynthesized low-cost QDs, would address DOE's National Goal of doubling energy productivity, dollars of gross domestic product (GDP) per unit of energy, by 2030. 


\section{TECHNICAL DISCUSSION OF WORK PERFORMED BY ALL PARTIES}

The research and development efforts conducted at ORNL were focused on 1) the development of postprocessing approach to suppress negative effects of surface defects in Nanofermented ZnS nanoparticles and 2) maximize photoluminescence efficiency of $\mathrm{ZnS}$ in aqueous solution. The second effort targeted application of post-processing treatment techniques (developed in first focus effort) to maximize

photoluminescence efficiency of $\mathrm{ZnS}$ nanoparticles produced at low temperature. The shelf-life of nanoparticles produced through post-treatment of nanoferemented $\mathrm{ZnS}$ (nf-ZnS) and low temperature chemically-synthesized $\mathrm{ZnS}(\mathrm{lts}-\mathrm{ZnS})$ nanoparticles was estimated.

Several postprocessing approaches to suppress negative effects of surface defects in nanofermented $\mathrm{ZnS}$ nanoparticles were tested, including:

Approach 1: Ligand passivation of nanoparticles, which is based on idea that the surface defects are commonly associated with uncompensated charge/vacancy on the surface of the nanoparticle, which could be used to attract ligands of opposite charge, and thus, reduce non-radiative relaxation and increase the efficiency of band gap emission.

Approach 2: Soft etching of the surface of nanoparticles. This approach is based on a photo-corrosion process, initiated by exposure of a nf-ZnS solution to high-flux ultra violet (UV) light. Upon excitation the non-radiative pathway for relaxation of the excited state would lead to increase in surface temperature of nanoparticle. This would lead to locally increased solubility of $\mathrm{ZnS}$ (at the solid-liquid interface), which would be the most active at the defect site. Higher concentration of $\mathrm{Zn}^{2+}$ and $\mathrm{S}^{-2}$ ions removed from the surface of $\mathrm{ZnS}$ nanoparticle create favorable condition for surface restructuring, where both ions could return to the surface, restoring the crystallinity of $\mathrm{ZnS}$. 


\subsection{CHEMICAL PASSIVATION OF SURFACE DEFECTS}

Surface modification of $\mathrm{nf}-\mathrm{ZnS}$ was carried using $\mathrm{Na}_{2} \mathrm{~S}$, urea and thiourea. The $\mathrm{nf}-\mathrm{ZnS}$ nanoparticles were exposed to $\mathrm{Na}_{2} \mathrm{~S}$, urea or thiourea for $12 \mathrm{~h}$ following centrifugation and washing cycles. The photoluminescence spectra were corrected to the values of corresponding absorbance at 320 and $350 \mathrm{~nm}$.
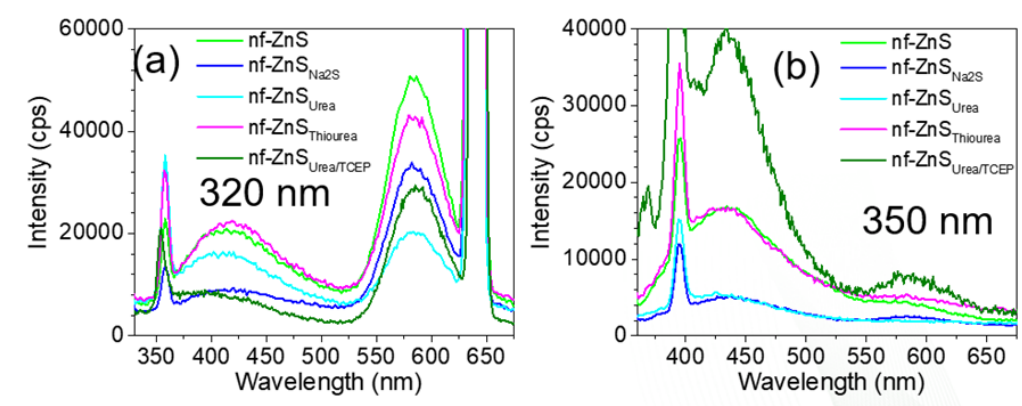

Figure 1 Effect of chemical treatment $\left(\mathrm{Na}_{2} \mathrm{~S}\right.$, urea and thiourea) on photoluminescence of $\mathrm{ZnS}$. The excitations was at (a) $320 \mathrm{~nm}$ and (b) $350 \mathrm{~nm}$.
The highest efficiency enhancement (4.5 times higher compared to untreated nf-Zn) was demonstrated (for $350 \mathrm{~nm}$ excitation) and urea/TCEP treated sample $\quad(\mathrm{nf}-\mathrm{ZnS}$ Urea/TCEP) at $\mathrm{pH}=7$, 12h). The results suggest that urea/TCEP passivate slow energy defect states, whereas higher energy states are almost unchanged as indicated by photoluminescence with $320 \mathrm{~nm}$ excitation. Chemical treatment at higher temperature $\left(60^{\circ} \mathrm{C}\right)$ or higher $\mathrm{pH}(\mathrm{pH}=10)$ led to a decrease in photoluminescence efficiency (for 320 and $350 \mathrm{~nm}$ excitation) and an increase in particle size. 


\subsection{PHOTOETCHING.}

The effect of extended UV irradiation on optical properties of $\mathrm{ZnS}$ (during the surface modification process) was investigated starting with as-synthesized $\mathrm{ZnS}$ nanoparticles in reactive media and exposed to fiber optic coupled UV light (404 nm, $75 \mathrm{~mW}$ for $3 \mathrm{~h}$ ) under continuous stirring. The reactive media was an aqueous solution, purged with nitrogen and adjusted to $\mathrm{pH}=10$ (by $0.1 \mathrm{M} \mathrm{NaOH}$ ). The nf-ZnS were rinsed/centrifuged 4x in DI $\mathrm{H}_{2} \mathrm{O}$ and $1 \mathrm{x}$ in $\mathrm{MeOH}$, and subsequently dispersed in $1 \% \mathrm{H}_{2} \mathrm{O}$ in $\mathrm{MeOH}$. Figure 2 below shows the effect of irradiation on optical properties of nf-ZnS. The samples were irradiated for $3.5,14$ and $24 \mathrm{~h}$ at $\mathrm{pH} 7,10$ and 12.
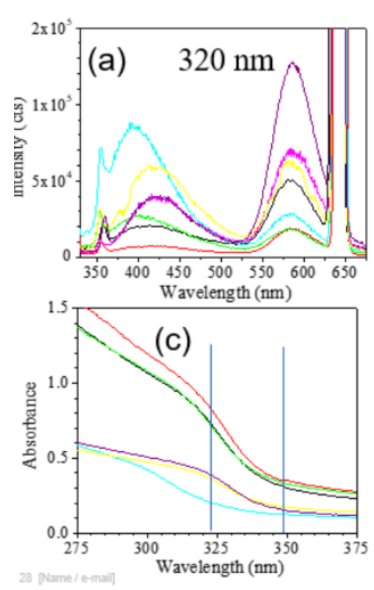

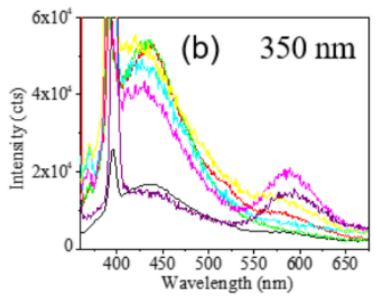

nf-ZnS RT, $\mathrm{pH}=07$ nf- $\mathrm{ZnS} 40^{\circ} \mathrm{C}, \mathrm{pH}=10$ $\mathrm{nf}-\mathrm{ZnS} \mathrm{pH}=07$ (1h UV post-wash) nf- $\mathrm{ZnS} \mathrm{pH}=07$ ( $3 \mathrm{~h}$ UV post-wash) nf- $\mathrm{ZnS} \mathrm{pH}=12$ (3h UV rxn) nf- $\mathrm{ZnS} \mathrm{pH}=07$, n2 purge (14h UV rxn) $\mathrm{nf}-\mathrm{ZnS} \mathrm{pH}=12$, $\mathrm{n} 2$ purge (24h UV rxn)

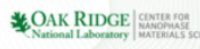

Figure 2 Effect of photoetching time and acidity on photoluminescence properties of $\mathrm{nf} \mathrm{ZnS}$ excited with $320 \mathrm{~nm}$ (a) and $350 \mathrm{~nm}$ (b). The spectra are normalized to the absorbance of nanoparticles at $320 \mathrm{~nm}$ and $350 \mathrm{~nm}$. High intensity line around $640 \mathrm{~nm}$ (c) is due to unfiltered second order Raleigh scattering. Vertical lines on panel $\mathrm{c}$ indicate excitation wavelength.

PL spectrum of nf-ZnS exhibits two strong broad emission bands at 420 and $590 \mathrm{~nm}$ with FWHM of about $50 \mathrm{~nm}$. The black-color lines of absorbance and photoluminescence spectra show properties of

as received nf-ZnS. Prolonged photoetching results in partial solubilization of nanoparticles, reducing the size of $\mathrm{nf}-\mathrm{ZnS}$ as evidenced by a shift in the edge of absorbance spectrum. The highest photoetching effect was observed at neutral $\mathrm{pH}=7$ and exposure of solution for $3 \mathrm{~h}$ to UV light. Normalization of PL spectra to the absorbance at excitation wavelength allows direct comparison of the PL spectra, the largest area under the spectrum indicates the material with the highest PL efficiency. We conclude that the $3 \mathrm{~h}$ and $12 \mathrm{~h}$ irradiate leads to the formation of the best material. The emission color characteristics of these materials are very different, the $3.5 \mathrm{~h}$ UV irradiated sample at neutral $\mathrm{pH}$ shows PL dominated by "blue" emission (320 nm excitation) while the $12 \mathrm{~h}$ sample with higher $\mathrm{pH}$ exhibits a mixed "blue- yellow" emission. The same pair of samples shows predominantly "blue" emission (maximum around $425 \mathrm{~nm}$ ) when excited at $350 \mathrm{~nm}$. These results suggest that the photo-etching reduced the density of shallow 
energy defects, increasing the photoluminescence efficiency almost by a factor of 2.5- 3 compared to asreceived $\mathrm{nf}-\mathrm{ZnS}$, for either 320 or $350 \mathrm{~nm}$ excitation.

\subsection{REACTIVE PHOTOETCHING.}

Modification of $\mathrm{pH}$ environment between neutral and $\mathrm{pH}=12$ lead to significant improvement in the photoluminescence intensity of $\mathrm{nf}-\mathrm{ZnS}$ nanoparticles. We further investigated possibility of photoetching combined with surface repair, which was provided by high concentration of repairing ions present in the etching environment. For surface repair during photoetching we used urea $\left(\mathrm{CH}_{4} \mathrm{~N}_{2} \mathrm{O}\right)$, thiourea $\left(\mathrm{CH}_{4} \mathrm{~N}_{2} \mathrm{~S}\right)$ and urea/TCEP binary system. The role of thiourea is to provide sulfide ions to $\mathrm{Zn}$ terminated nanoparticles, thus repairing the ion vacancy. Tris(2-carboxyethyl) phosphine hydrochloride (TCEP) is a strong reducing agent which is also used to break disulfide bonds (possible defects or residual of protein
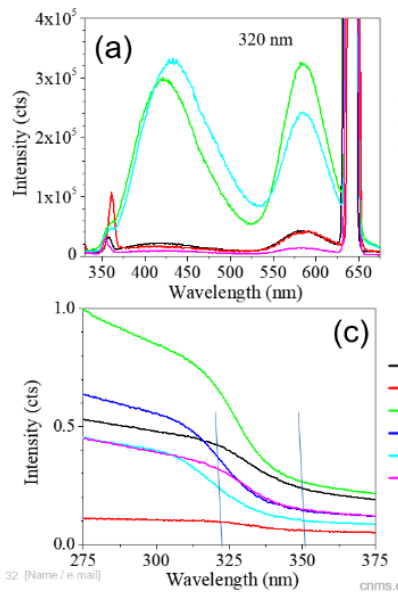

)
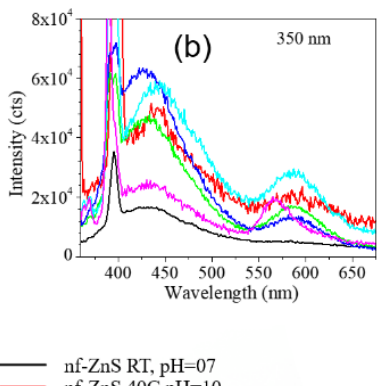
nf- $\mathrm{ZnS} 40 \mathrm{C}, \mathrm{pH}=10$ nf- $\mathrm{ZnS} 40 \mathrm{C}, \mathrm{pH}=10$ (1h UV post-wash) $\mathrm{nf}-\mathrm{ZnS} 40 \mathrm{C}, \mathrm{pH}=10(2 \mathrm{~h}$ UV post-wash)
$\mathrm{nf}-\mathrm{ZnS} 40 \mathrm{C}, \mathrm{pH}=10$ ( $3 \mathrm{~h}$ UV post-wash) nf-ZnS RT pH=07 (3h UV rxn)

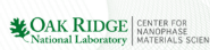

bound to the surface of nf-ZnS). Urea is used as protein denaturant (for surface cleaning of $\mathrm{nf}-\mathrm{ZnS}$ ).

The figures below demonstrate the results of reactive photoetching. The results of reactive photoetching are shown in figure 3, below.

Figure 3. Changes in optical properties of reactively photoetched nf-ZnS with thiourea. (a) absorption corrected photoluminescence generated using $320 \mathrm{~nm}$ excitation, (b) absorption corrected photoluminescence obtained with $350 \mathrm{~nm}$ excitation.

It is apparent, that for $320 \mathrm{~nm}$ excitation the best improvement in photoluminescence efficiency was the factor of 20 increase, compared to as-received $\mathrm{ZnS}$, observed for $\mathrm{nf}-\mathrm{ZnS}$ treated by thiourea and exposed to $\mathrm{UV}$ for $1-3 \mathrm{~h}$ at $\mathrm{pH}=10$ and $\mathrm{T}=40^{\circ} \mathrm{C}$. It is interesting to note that improvement of PL efficiency for excitation with $350 \mathrm{~nm}$ was only a factor of 3 , suggesting that excess of $\mathrm{S}^{-2}$ ions under photoetching conditions does not allow complete removal of defects. The presence of the $580 \mathrm{~nm}$ emission band in treated samples further supports that additional defects associated with excess of $\mathrm{S}^{-2}$ may be produced. When nf-ZnS was exposed to UV light photoetching in the presence of $\mathrm{Na}_{2} \mathrm{~S}, \mathrm{pH}=10$ and $\mathrm{T}=40^{\circ} \mathrm{C}$, an 
improvement by a factor or 15 was demonstrated for samples treated for 1 and 3 hours (considering 320 $\mathrm{nm}$ excitation as a reference point), Figure 4.
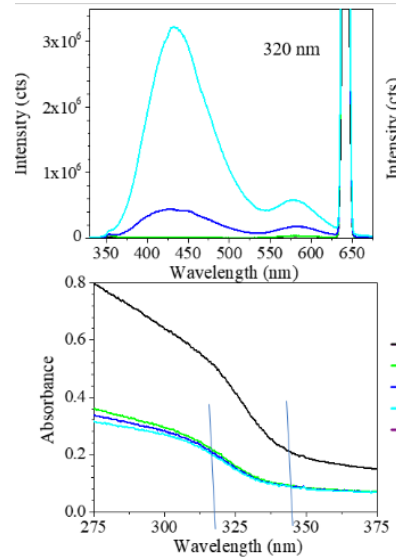

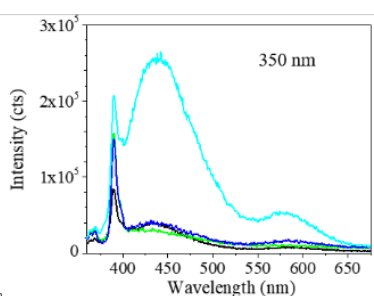

- nf-ZnS RT RT, $\mathrm{pH}=10$

nf-ZnS RT, $\mathrm{pH}=10$ (1 h UV post-wash)

nf-ZnS RT, $\mathrm{pH}=10$ ( $3 \mathrm{~h}$ UV post-wash) nf- $\mathrm{ZnS} \mathrm{pH}=10$ (24h UV post-wash)

* OAK RIDGE Gaviarege
Figure 4. Effect of reactive photoetching in the presence of $\mathrm{Na}_{2} \mathrm{~S}$ on optical properties of $\mathrm{nf}-\mathrm{ZnS}$. The photoluminescence of $\mathrm{nf}-\mathrm{ZnS}$ was normalized to the absorbance at the excitation wavelength.

For both samples the intensity of emission at $420 \mathrm{~nm}$ and $570 \mathrm{~nm}$ is about the same. For samples excited at $350 \mathrm{~nm}$ the improvement in the photoluminescence efficiency is smaller, but the band gap fluorescence

shows double the efficiency than that with only the defect originated emission at $570 \mathrm{~nm}$. While photoetching at basic $\mathrm{pH}$ in the presence of $\mathrm{Na}_{2} \mathrm{~S}$ indeed improved the photoluminescence properties of asreceived $\mathrm{nf}-\mathrm{ZnS}$, without photoetching the bandgap photoluminescence is completely quenched and only defect related PL is observed. This observation allows us to make a conclusion that excess of $\mathrm{S}^{-2}$ ions completely covers the surface of the $\mathrm{ZnS}$ nanoparticle, however, and this excess also creates a pathway for non-radiative relaxation of the excited states. The absorbance spectra show slight increase at high
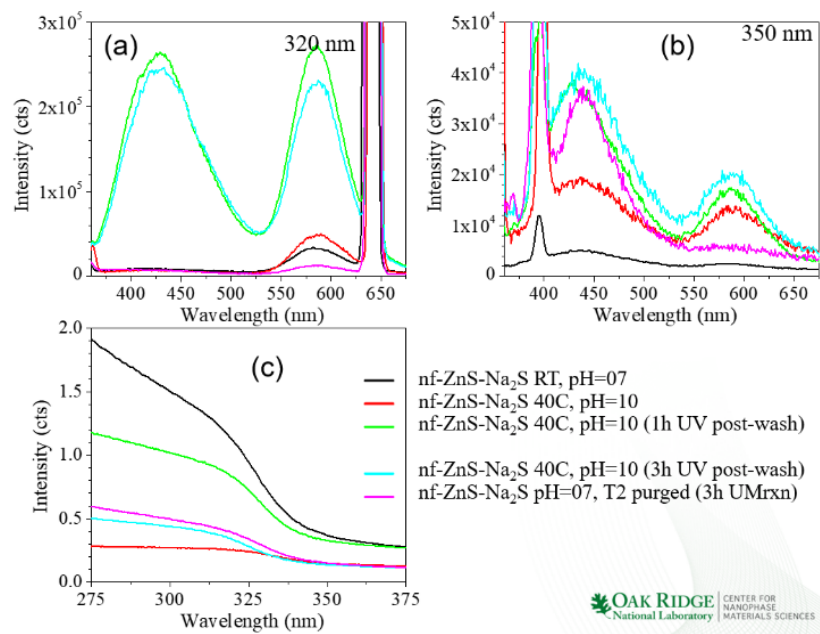

nf-ZnS- $\mathrm{Na}_{2} \mathrm{~S} \mathrm{RT}, \mathrm{pH}=07$ nf-ZnS-Na 2 S $40 \mathrm{C}, \mathrm{pH}=10$

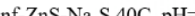
nf-ZnS- $\mathrm{Na}_{2} \mathrm{~S} \mathrm{pH}=07$, T2 purged (3h UMrxn) * OAK RIDGE |comrarog energy part of the spectrum.

Figure 5. The effect of photoetching in basic media $(\mathrm{pH}=10)$, urea/TCEP at room temperature.

Low temperature photoetching of $\mathrm{nf}-\mathrm{ZnS}$ in ureaTCEP media requires 2-3 hours to reach highest enhancement of photoluminescence properties (by a factor of 4), predominantly improving band gap emission, (Fig. 5). The photoetching also reduces the absorption at wavelengths longer than

the band gap, the observation is concomitant with reduction in defect-originated luminescence. 
The effect of irradiation time (1, 2, 3, 4, and $24 \mathrm{~h})$ on the efficiency of photoluminescence was studied for urea and thiourea modified in the presence of TCEP. Photoluminescence observed with $320 \mathrm{~nm}$ excitation indicates that irradiation of urea/TCEP for longer than $3 \mathrm{~h}$ does not result in additional improvement beyond those observed for $3 \mathrm{~h}$ irradiation. However, with thiourea, the best photoluminescence was observed after $24 \mathrm{~h}$ irradiation.

In previous experiments we observed changes on the band gap of $\mathrm{ZnS}$, indicating that the diameter of nanoparticles reduced. We therefore tested the higher energy excitation $(300 \mathrm{~nm})$ to probe photoluminescence of smaller particles, which are expecting to absorb at much higher energy.
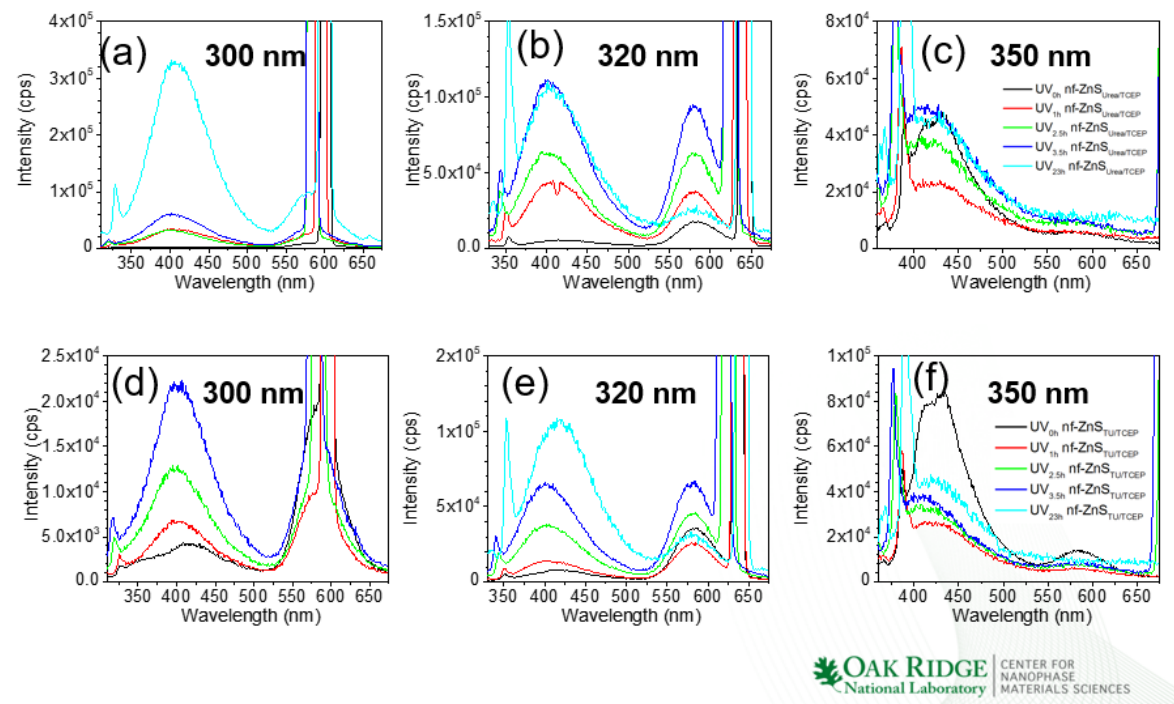

Figure 6. Normalized photoluminescence of photoetched of urea/TCEP modified nf-ZnS (chemically modified in $\mathrm{N}_{2}$ purged $\mathrm{H}_{2} \mathrm{O}$, RT for $16 \mathrm{~h}$, rinsed/centrifuged $3 \mathrm{x}$ in $\mathrm{H} 2 \mathrm{O}$ and $1 \mathrm{x}$ in methanol, then re-dispersed in $1 \% \mathrm{H}_{2} \mathrm{O}$ in methanol. UV-a under air at $404 \mathrm{~nm}, 75 \mathrm{~mW}$ in $1 \% \mathrm{H}_{2} \mathrm{O}$ in methanol) and excited with (a) $300 \mathrm{~nm}$, (b) $320 \mathrm{~nm}$ and (c) $350 \mathrm{~nm}$. Normalized photoluminescence of photoetched treatment of thiourea/TCEP modified nf- ZnS (chemically modified in $\mathrm{N}_{2}$ purged $\mathrm{H}_{2} \mathrm{O}$, room temperature for $16 \mathrm{~h}$, rinsed/centrifuged $3 \mathrm{x}$ in $\mathrm{H}_{2} \mathrm{O}$ and $1 \mathrm{x}$ in methanol, then re-dispersed in $1 \% \mathrm{H}_{2} \mathrm{O}$ in methanol. UV-irradiation under air at $404 \mathrm{~nm}, 75 \mathrm{~mW}$ in $1 \% \mathrm{H}_{2} \mathrm{O}$ in methanol.)

Figure 6, panels (a) and (d) show the effect of $16 \mathrm{~h}$ photoetching with urea/TCEP and $3 \mathrm{~h}$ irradiation in the presence of thiourea/TCEP on photoluminescence properties of nf-ZnS. We observed that the nanoparticles excited at $350 \mathrm{~nm}$ show predominantly bandgap emission, with minimum defect photoluminescence. Interestingly, the best photoluminescence at $350 \mathrm{~nm}$ excitation was observed for thiourea/TCEP without irradiation. It suggests a possible low-cost pathway to modify nanoparticles without expense associated 
with implementation of UV etching system. The summary of the optical properties of $\mathrm{nf}-\mathrm{ZnS}$ nanoparticles exposed to a different photoetching time are shown in Table. They demonstrate that a factor of 5 improvement can be achieved after $1 \mathrm{~h}$ of treatment.

Table 1. A summary of optical properties of nf-ZnS nanoparticles which underwent photoetching postprocessing treatment.

\begin{tabular}{|l|c|c|c|c|c|c|c|}
\hline & UV Time & $\varepsilon_{320 \mathrm{~nm}}$ & $\mathrm{~A}_{320 \mathrm{~nm}}$ & $\mathrm{~F}_{\mathrm{x}}\left(\int_{\text {Corr }}\right)$ & $f_{\mathrm{x}}$ & $\mathrm{F}_{\mathrm{x}} / f_{\mathrm{x}}$ & $\Phi_{\mathrm{x}}(\%)$ \\
\hline \multirow{3}{*}{$\mathrm{nf}-$} & 0 & 341.8 & 0.731 & 1.239 & 0.814 & 1.521 & 0.50 \\
\cline { 2 - 8 } $\mathrm{ZnS}$ & $1 \mathrm{~h}$ & 226 & 0.484 & 5.073 & 0.672 & 7.549 & 2.50 \\
\cline { 2 - 8 } & $2 \mathrm{~h}$ & 140 & 0.301 & 0.525 & 0.500 & 1.051 & 0.34 \\
\cline { 2 - 8 } & $2 \mathrm{~h}+3 \mathrm{~d}$ & 92 & 0.197 & 0.451 & 0.364 & 1.237 & 0.41 \\
\cline { 2 - 8 } & $2 \mathrm{~h}+4 \mathrm{~d}$ & 86 & 0.180 & 0.466 & 0.340 & 1.372 & 0.45 \\
\hline
\end{tabular}




\subsection{CONTROL OF THE SURFACE DEFECT DENSITY IN LOW TEMPERATURE SYNTHESIS OF ZNS NANOPARTICLES.}

We demonstrated that the surface defects of $\mathrm{nf}-\mathrm{ZnS}$ nanoparticles can be partially removed through a carefully controlled photoetching in the presence of reducing or surface passivation agents. However, the applicability of the approach is limited to the surface defects of nanoparticle which are exposed to the liquid media and modification agents, and it does not affect defects embedded within the nanoparticle structure. The excess of surface material needs to be etched off to access buried defects (which may be within a

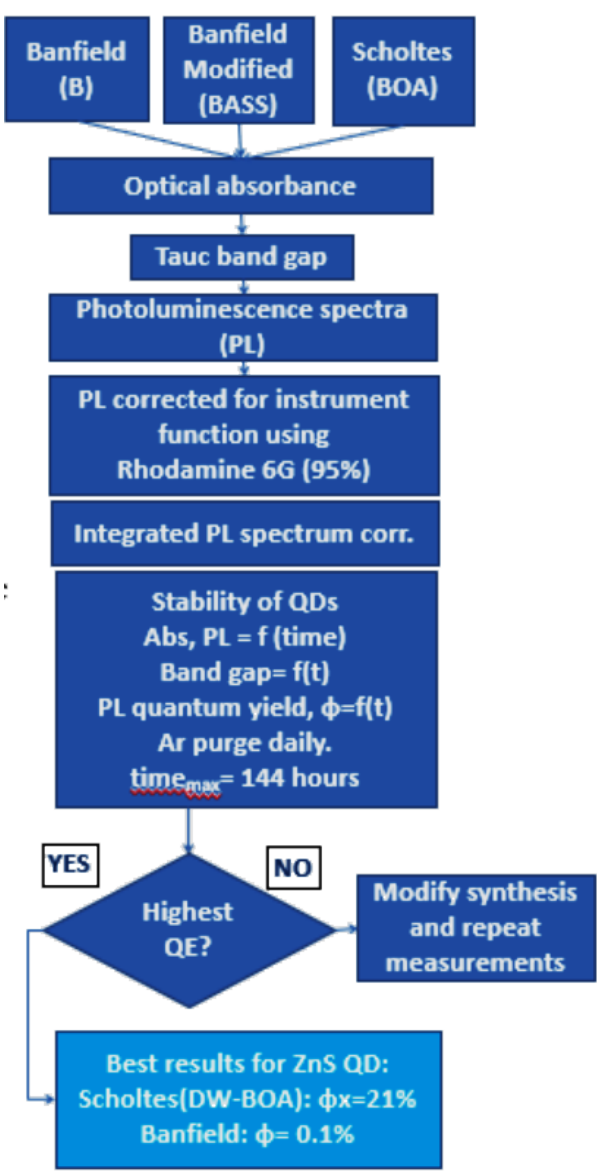
monolayer from the surface). Such etching may reduce the size of the nanoparticles and therefore shift the emission of nanoparticles to the higher energy, making them less suitable for solid state light applications. Implementation of the surface passivation during the low temperature synthesis of nanoparticle could potentially allow mitigation of defects during synthesis of nanoparticles. The nanoparticle growth may be slowed because surface passivation may render some area of particle inactive. However, slow growth of nanoparticle of higher quality may be economically more advantageous compared to the cost of post-treatment processing of nf- $\mathrm{ZnS}$.

Figure 7 Experimental workflow used for chemical synthesis of ZnS targeted the development nanoparticles with the highest photoluminescence efficiency

ZnS QD nanoparticles were prepared in the media of anhydrous methanol $(\mathrm{MeOH})$ by combining $1 \mathrm{mM}$ sodium sulfide $\left(\mathrm{Na}_{2} \mathrm{~S}\right)$ and $1 \mathrm{mM}$ zinc chloride $\left(\mathrm{ZnCl}_{2}\right)$ under inert $\mathrm{Ar}$ atmosphere, while under continuous sonication ${ }^{(5)}$. Oleic acid (OA) was selected as the primary capping agent to reduce oxidation of nanoparticle surface ${ }^{(1,2)}$. Samples identified as $B A S S$ and $B O A$ were synthesized with and without oleic acid surfactant respectively. The samples marked as $\mathrm{Zn} 3 \mathrm{x}$ contained an excess of $\mathrm{Zn}$ in respect to sulfur. Samples were centrifuged at 10,000 rpm before sonication or vortex spinning. Shelf life of samples was measured daily, keeping samples under 
argon atmosphere. The PLQE quantum yield $\left(\phi_{\mathrm{F}}\right)$ of re-dispersed OA-nf ZnS colloid was determined by comparative methods using a solution of Rhodamine B with matching optical density at excitation wavelength as a standard. The $\left(\phi_{\mathrm{F}}\right)$ of OA-nf $\mathrm{ZnS}$ was found to be $5.8 \%$. Band gap of $\mathrm{ZnS} /$ bio $\mathrm{ZnS}$ was estimated to be $4.1 \mathrm{eV}$. Optical propertied of the metal sulfide nanoparticles (absorbance and photoluminescence) were measured.

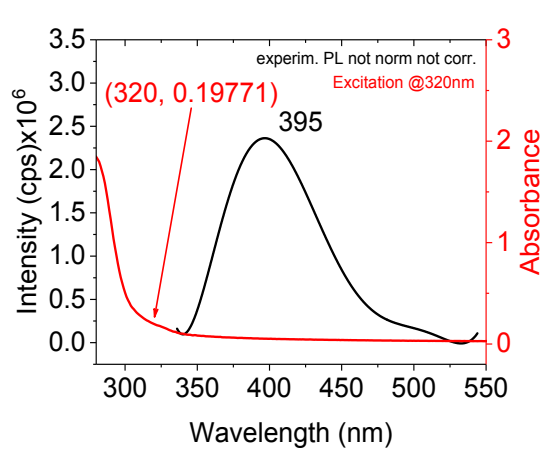

Figure 9. Optical properties of ZnS QD's prepared with DW- OA synthesis. Absorbance (red) and photoluminescence spectrum for $320 \mathrm{~nm}$ excitation showing the emission peak at $395 \mathrm{~nm}$. Photoluminescence normalized to absorption at excitation wavelength is shown on the right panel.

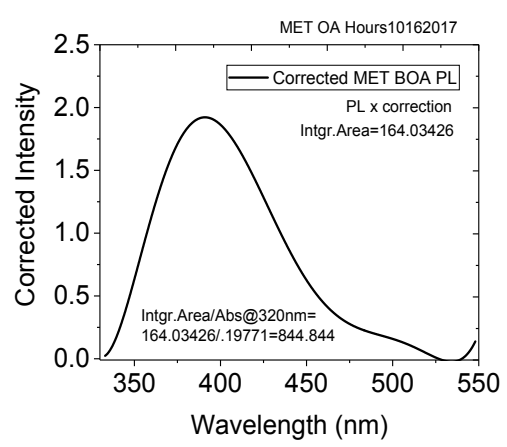

first. Then, $50 \mathrm{ml}$ stock solutions of $1 \mathrm{mM}$ of $\mathrm{ZnCl}_{2}$ in methanol $(\mathrm{MeOH})$ and

the $1 \mathrm{mM}$ of $\mathrm{Na}_{2} \mathrm{~S}$ in methanol $(\mathrm{MeOH})$ were prepared. The stoichiometric mixture of $\mathrm{Na}_{2} \mathrm{~S}$ was added into the $\mathrm{ZnCl}_{2}$ under inert atmosphere (Ar bubbled through a septum in a round bottom flask for 15 minutes).
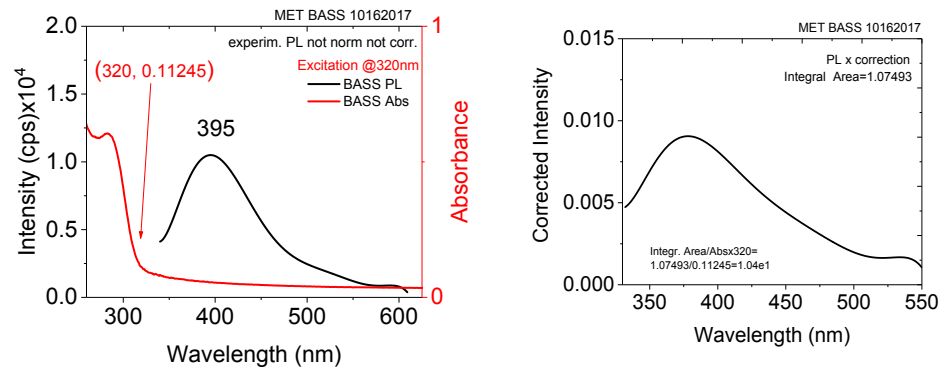

The $1 \%$ vol. OA was added to the solution as $\mathrm{Na}_{2} \mathrm{~S}$. Zn3x sample (ratio of $3: 1 \quad \mathrm{Zn}: \mathrm{S}$ ) was synthesized using method mentioned above.

Figure 8.DW ZnS BASS. Absorbance excited at 320nm and Photoluminescence emission at $395 \mathrm{~nm}$ and corrected photoluminescence to the right.

Synthesis of $\quad \mathrm{Zn} 3 x-\mathrm{C} / \mathrm{S}$

centrifugation \& sonication: we used $1 \mathrm{mM}$ stock solutions of $\mathrm{ZnCl}_{2}$ degassed with Ar for 5 minutes before synthesis. Samples were centrifuged at 10,000 rpm for 15 minutes after nanoparticle synthesis. An excess of $\mathrm{MeOH}$ extracted with equivalent volume of $\mathrm{MeOH}$ then quickly purged with Ar before sonication at 
100\% power (Ingenieurbüro CAT, M. Zipperer GmbH) (Type: X120 130W 50/60Hz) at a distance of 5cm for 3 minutes. This sonication cycle was repeated three times.

Synthesis using drop-wise (DW) ZnS: OA (1\% vol.) $1 \mathrm{mM} \mathrm{ZnCl}_{2}$ stock solution was degassed with Ar for 5 minutes. Then a $1 \mathrm{mM} \mathrm{Na} 2 \mathrm{~S}$ solution under Ar was slowly added at $1 \mathrm{ml} / \mathrm{min}$ dropwise to the $\mathrm{ZnCl}_{2} / \mathrm{OA}$ mixture under a smooth stir (maintained while dropwise addition) and bubbled Ar for 15 minutes.

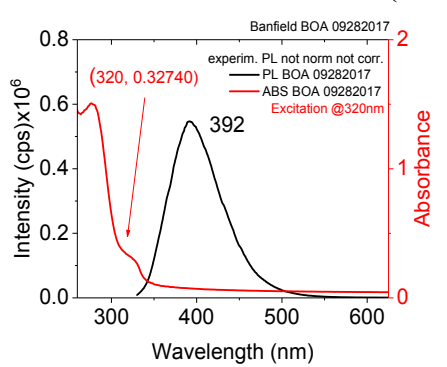

Figure 10. Optical properties of ZnS nanoparticles synthesized using BOA. Absorbance and photoluminescence produced by $320 \mathrm{~nm}$ excitation with a maximum at $395 \mathrm{~nm}$. The absorbance- photoluminescence is shown on the right panel.

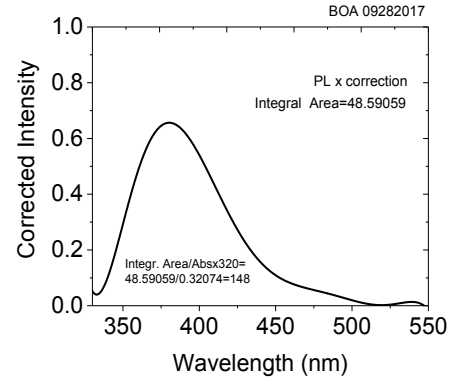

$\mathrm{ZnS}$ BOA was over an order magnitude higher than that of $\mathrm{Zn} 3 \mathrm{x}$ $\mathrm{C} / \mathrm{S}$ sample and the stability after 144

hours was also higher compared to the normalized PL intensity of $\mathrm{Zn} 3 \mathrm{x} \mathrm{C} / \mathrm{S}$. The photoluminescence of DW ZnS BOA sample stabilized after 24 hours.

The DW ZnS BASS sample had better stability over the Zn3x C/S sample, yet the value of absorbancenormalized PL intensity was lower. The DW method produced significant results in the BOA sample, yielding the highest normalized PL and QY for all the BOA samples. We modified the original Banfield synthesis to run it under argon, and showed that while the absorbance of DW ZnS N${ }_{2}$ BASS and BOA samples was the same, the photoluminescence of samples produced under $\mathrm{N}_{2}$ atmosphere was much lower than that produced under argon. Synthesis in an excess of $\mathrm{Zn}^{2+}$ ions led to a decrease in the normalized PL, most likely due to formation of amorphous $\mathrm{ZnS}$ clusters in the BASS and BOA samples. Increasing the concentration of OA from $1 \%$ to $5 \%$ led to an increase in the stability of the sample. The DW ZnS BOA $10 \mathrm{mM}$ at $5 \%$ OA displayed an order of magnitude higher efficiency after 48 hours compared to the same sample with $1 \%$ OA. The absorbance of DW ZnS 10mM BASS sample was about 1.159 compared to 0.113 
in the DW ZnS BASS sample. The absorbance of the DW ZnS 10mM BOA sample was 0.875 compared to 0.196 in the DW ZnS BOA sample. Table 2 summarizes optical properties of chemically synthetized $\mathrm{ZnS}$ nanoparticles by all methods mentioned above, including band gap information and efficiency of photoluminescence. The highest efficiency of $\mathrm{ZnS}$ fluorescence $(21 \%)$ was obtained in DW- BOA synthesis, this is a factor of 5 higher than the DW-BASS approach.

Table 2 Optical properties of chemically synthetized $\mathrm{ZnS}$ nanoparticles

\begin{tabular}{|l|c|c|c|c|}
\hline & \multicolumn{2}{|c|}{ BASS } & \multicolumn{2}{c|}{ BOA } \\
\hline Synthesis & $\phi \mathrm{x}$ & Bandgap $(\mathrm{eV})$ & $\phi \mathrm{x}$ & Bandgap $(\mathrm{eV})$ \\
\hline ZnS & 0.04 & 4.06 & 3.74 & 4.10 \\
\hline Zn3x & 1.06 & 4.17 & 2.33 & 4.10 \\
\hline Zn3x-C/S & 2.11 & 4.20 & 1.69 & 4.09 \\
\hline DW ZnS N2 & 0.1 & 4.03 & 5.33 & 4.08 \\
\hline DW ZnS & 0.26 & 4.01 & 20.91 & 4.04 \\
\hline DW ZnS Sonic & 0.15 & 4.21 & 6.41 & 4.18 \\
\hline DW ZnS UV & 0.23 & 4.24 & 0.61 & 4.12 \\
\hline
\end{tabular}

$* \phi x$ values compared to photoluminescence efficiency of Rhodamine $6 \mathrm{G}(95 \%)$

It is interesting to note that the bandgap (and the size of nanoparticles) of $\mathrm{ZnS}$ prepared by DW-BOA and DW-BASS methods are about the same, and their band gap is about $4.0 \mathrm{eV}$. The second to the best DW$\mathrm{ZnS}$ efficiency of about $20.91 \%$ was the efficiency of DW-ZnS-sonic (6.4\%). 


\subsection{INVESTIGATION OF THE STABILITY OF CHEMICALLY SYNTHESIZED ZNS NANOPARTICLES}

The stability of chemically synthesized $\mathrm{ZnS}$ nanoparticles was studied by following changes in absorbance and photoluminescence over 144 hours while keeping solutions under inert atmosphere. All nanoparticles produced by BOA, BASS, Zn3x-BASS and Zn3x-BOA approaches showed rapid decline of photoluminescence over the first 24 hours, followed by stabilization of photoluminescence. After the first decline, the efficiency of luminescence of Zn3x nanoparticles did not change over the $144 \mathrm{~h}$ period, whereas nanoparticles obtained through the BASS and BOA approach showed a second decline in efficiency after $120 \mathrm{~h}$.

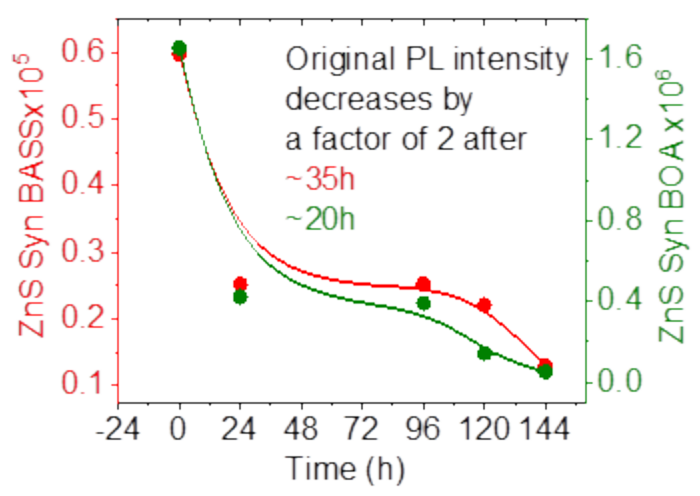

(a)

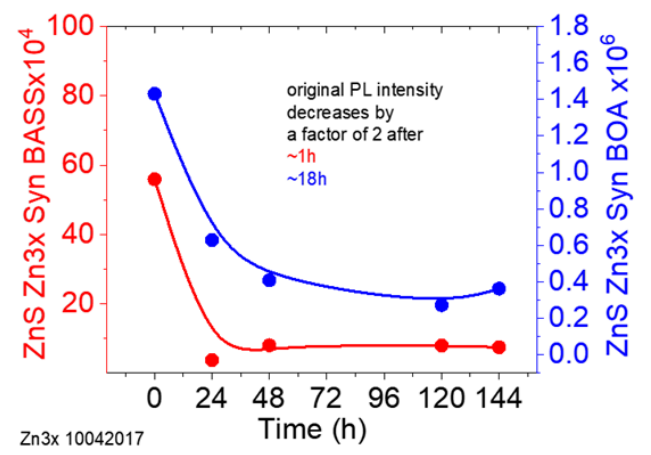

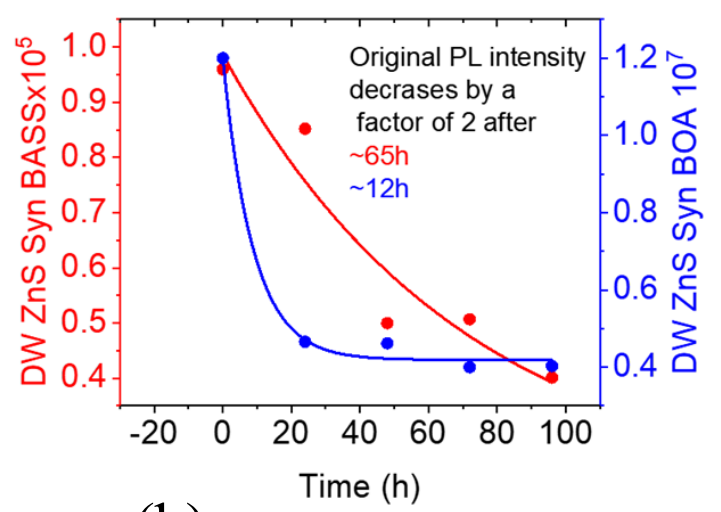

(b)

Figure 11. Stability of chemically synthesized $\mathrm{ZnS}$ prepared using different methods. (a) Changes in the intensity of normalized photoluminescence (392 nm peak) to absorbance (at $320 \mathrm{~nm}$ ) of chemically synthesized $\mathrm{ZnS}$ in $\mathrm{MeOH}$ purged with argon under excited. (b) Changes in the intensity of normalized photoluminescence to absorbance (395nm peak) of synthesized DW ZnS in $\mathrm{MeOH}$ purged with argon excited $320 \mathrm{~nm}$ over 144 hours. (c) Changes in the intensity of normalized photoluminescence (392nm peak) of synthesized $\mathrm{ZnS}$ ( $\mathrm{Zn}: \mathrm{S}$ 3:1 ratio) in $\mathrm{MeOH}$ purged with argon excited $320 \mathrm{~nm}$ over a 144 hours.

The PL intensity of DW-ZnS BASS decreased by a factor of 2 over $\sim 65 \mathrm{~h}$ while the PL intensity of DWBOA ZnS decreased by a factor of 2 over first 12 hours, and then stabilized at about $40 \%$ of original value. Samples of $\mathrm{ZnS}$ obtained in $3 \mathrm{x}$ excess of $\mathrm{Zn}^{2+}$ showed relatively similar stability with the PL intensity 
decreasing over first $24 \mathrm{~h}$ by a factor of 2 . Thus, depending on application, the tradeoff high efficiency and long shelf life of $\mathrm{ZnS}$ nanoparticles should be considered. UV photo etching was used during synthesis and for post processing of chemically synthesized $\mathrm{ZnS}$, resulting in a higher rate of PL intensity reduction for the BOA samples, compared to the BASS samples, as seen in table below 3. The post processing photoetching of chemically synthetized ZnS BASS lead to increase in sample absorption after 45 minutes, while absorbance of $\mathrm{ZnS}$ BOA samples did not increase of the same time. The absorbance $\mathrm{Zn} 3 \mathrm{x}$ BOA samples was higher compared with the $\mathrm{ZnS}$ BOA samples, while the absorbance of the Zn3x BASS and ZnS BASS samples decreased in a comparable manner.

Sonication- assisted synthesis of ZnS. DW-ZnS-sonic-BASS samples displayed absorbance comparable to DW ZnS samples, however, the absorption-normalized photoluminescence values were lower than those of the DW ZnS Sonic BASS and BOA samples.

Table 3. Normalized photoluminescence to absorption at 320nm. (Photoluminescence intensities are divided by 1000).

\begin{tabular}{|l|c|c|c|c|c|c|}
\hline Synthesis & \multicolumn{3}{|c|}{ BASS } & \multicolumn{3}{c|}{ BOA } \\
\hline Processing & No UV & 15 min. & 45 min. & No UV & 15 min. & 45 min. \\
\hline ZnS UV & 18 & 14 & 6 & 3190 & 2130 & 180 \\
\hline Zn3x UV & 220 & 64 & 11 & 4700 & 3920 & 290 \\
\hline
\end{tabular}




\subsection{COST ANALYSIS}

NanoFermentation of $\mathrm{ZnS}$ quantum dots costs $\$ 0.067$ per gram. This is in sharp contrast to commercial quantum dots which cost $\sim \$ 200$ per gram. The majority of this cost, $83 \%$, is the media required to grow the bacteria while nanoparticle precursors are $8 \%$ and equipment is $5 \%$. The post processing improvements described here only add $1.5 \%$ to the cost of $\mathrm{ZnS}$ quantum dots while greatly improving their value to the market. With these improvements nanofermented $\mathrm{ZnS}$ quantum dots remain an economically viable option. 


\subsection{MARKET FIT.}

The most lucrative market for quantum dot is next generation of phosphor for display technology. The technical requirements of quantum dots for this market are defined by purity and quality of color and the efficiency of electroluminescence along with the stability of their optical properties under application conditions. Some specific properties include red emitter emission maximum at $\sim 620 \mathrm{~nm}(20 \mathrm{~nm}$ FWHM), and green emitter with maximum at $520 \mathrm{~nm}(20 \mathrm{~nm})$ FWHM). The efficiency of luminescence should exceed $75 \%$ and preferably approaching $90 \%$. The quantum dots are expected to perform without degradation of the properties over 10,000 hours under elevated temperature of $100^{\circ} \mathrm{C}$ in the film. 


\subsection{COMMERCIALIZATION POSSIBILITIES}

Production of high photoluminescent efficiency $\mathrm{ZnS}$ nanoparticles with low cost techniques demonstrated in this project opens several commercialization opportunities.

1) The technology verified in this project allows for the improvement of nf- $\mathrm{ZnS}$ photoluminescence efficiency by a factor of 20 , using the post-processing approach. This technology can potentially be extended to other composition of nanoparticles produced through NanoFermentation.

2) Commercialization of $\mathrm{ZnS}$ QDs as a phosphor screen in electronic devices. We have demonstrated that $\mathrm{ZnS}$ nanoparticles (modified $\mathrm{nf}-\mathrm{ZnS}$ and chemically synthetized $\mathrm{ZnS}$ ) can be deposited on the surface of quartz/glass forming stable coatings.

3) Commercialization of $\mathrm{ZnS} \mathrm{QD}$ as antibacterial and antifungal components. The technology was recently disclosed (ORNL IDEA) and demonstrated in collaboration with Texas AM medical center. (manuscript accepted for publication).

4) Chemical synthesis of $\mathrm{ZnS}$ nanoparticles with efficiency above $20 \%$ can be implemented using $\mathrm{H}_{2} \mathrm{~S}$ outgas from the NanoFermentation process (ORNL disclosure, patent application) 


\subsection{PLANS FOR FUTURE COLLABORATION}

The results of R\&D efforts in this project clearly indicate the possibility to design low-cost technology of high efficiency luminescent nanoparticles, which can be applied to other nanoparticle compositions. Several opportunities have been identified to further the nanoparticle technology through future collaboration.

The first opportunity is to use the procedures demonstrated here to lower the cost and increase the photoluminescence efficiency of the quantum dots/nanoparticles which are currently used as LED phosphors. There include core-shell nanoparticles with different composition of core (i.e. CdS) and shell

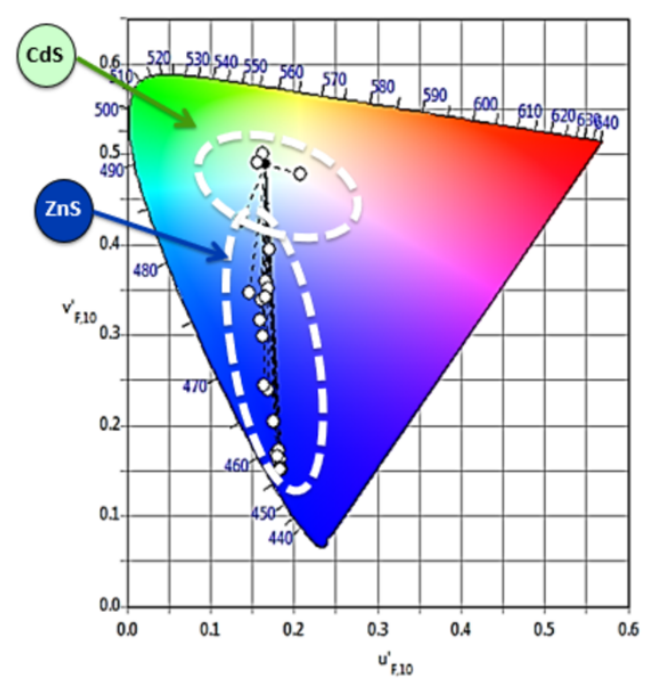

Figure 12. Chromaticity diagram showing broad range of photoluminescence colors (from blue to white) which can be produced in CdS: $\mathrm{ZnS}$ cocolloids. made of $\mathrm{ZnS}$. The chemical synthesis procedures developed and verified in this project allow production of low defects of $\mathrm{ZnS}$ shell, thus creating a potential to further improve efficiency of photoluminescence of core-shell nanoparticles.

The second opportunity includes incorporation of the technology developed in this project for production of green, red and white-color QD suitable for application in solid state lighting devices. Our preliminary investigation (conducted as part of CNMS fundamental research program) demonstrated one possible strategy to produce $\mathrm{CdS}: \mathrm{ZnS}$ co-colloids, the

emission color of which can be controlled by changing the ratio of $\mathrm{CdS}$ and $\mathrm{ZnS}$ components. Figure 12 demonstrates the color quality of broad range of $\mathrm{CdS}: \mathrm{ZnS}$ co-colloids generated in our laboratory (open circles) on the human color perception map (in terms of two CIE parameters $\mathrm{x}$ and $\mathrm{y}$ ). The chromaticity diagram is designed to display objective specification of the quality of a color independent of it luminance (intensity). Further development may include demonstration of green and red- luminescent particles.

The third opportunity involved implementation of optimized chemical synthesis using outgas from the fermentation chamber. The concept of this technology was recently disclosed and patented by ORNL (Ji Won Moon et. all). There, the $\mathrm{H}_{2} \mathrm{~S}$ outgassed from the fermentation can be used to synthesized quantum 
dots or nanoparticles, including those which present commercial interest, as mentioned above. We believe that this opportunity presents the shortest commercialization route out of three opportunities mentioned above.

Based on our contacts with other companies, future research could target application of quantum dots for new markets (including lighting). With focused R\&D efforts on the development \& enhancement of core shell structures, and integration with the improved $\mathrm{ZnS}$ quantum dots described here, NanoFermented quantum dots could be optimized for horticultural lighting applications (with peak emission at $470 \mathrm{~nm}$ and $660 \mathrm{~nm}$ ). Additionally, quantum dots that demonstrate fast switching speeds between their on and off states would be ideal for enhanced data transfer. Of particular interest is the development of stable UV emitting QDs for UV sterilization. 


\section{CONCLUSIONS}

We investigated a broad range of techniques to improve efficiency of $\mathrm{nf} \mathrm{ZnS}$ nanoparticles and provided several routes to improve their photoluminescence efficiency through postprocessing. We also concluded that even higher efficiencies can be achieved for nanoparticles synthetized under strict environment of chemical synthesis. We also showed that postprocessing does not increase the cost of nf-Zn, keeping them a low- cost material comparing with current commercial cost of $\mathrm{ZnS}$. 


\section{ACKNOWLEDGMENTS}

This research was conducted through CNMS2017- 164 user research proposal at the Center for Nanophase Materials Sciences, which is a DOE Office of Science User Facility. Nanofermented ZnS nanoparticles for the project were synthesized by Dr. Ji-Won Moon. We acknowledge Dr. Chris Rouleau help with the CNMS2017-164 user research project. 